\title{
DETERMINATION OF THE TENSILE RESPONSE OF UHPFRC LAYERS USING A NON-DESTRUCTIVE METHOD FOR ASSESSING THE FIBER CONTENT AND ORIENTATION
}

\author{
MÁRIO PIMENTEL ${ }^{*}$, SANDRA NUNES ${ }^{\dagger}$ \\ University of Porto, Faculty of Engineering \\ Porto, Portugal \\ e-mail: mjsp@fe.up.pt \\ ${ }^{\dagger}$ University of Porto, Faculty of Engineering \\ Porto, Portugal \\ e-mail: snunes@fe.up.pt
}

\begin{abstract}
A procedure is developed for estimating the representative tensile response of existing thin Ultra-High Performance Fiber-Reinforced cement Composite (UHPFRC) layers. The procedure relies on non-destructive (NDT) measurements and on a previous tensile characterization campaign of the composite material, thereby avoiding the extraction of samples from the real structure. Two reliable indicators of the fiber content and fiber orientation within the UHPFRC layer are derived from the NDT measurements along two orthogonal directions. It is shown that these two indicators can be directly correlated to the parameters of a mechanical model providing the tensile response of the composite. The procedure is applied for the prediction of the peak stress of 36 Double-Edge Wedge Split tests and of the structural behavior of slab strips strengthened with a UHPFRC layer.
\end{abstract}

Keywords: Ultra-high performance fiber-reinforced cement-based composites (UHPFRC), tensile response, fiber orientation, magnetic method, non-destructive testing (NDT)

\section{INTRODUCTION}

Besides local variations of the fiber content, the tensile response of UHPFRC varies significantly according to fiber orientation [1][2][3], which depends on the placement process, on the rheological behaviour of the material in the fresh state, and on the geometric shape of the form to be cast. This means that an intrinsic tensile response for UHPFRC cannot be defined, and the representative tensile response must be used instead in order to achieve reliable estimates of the structural behavior.

In general, the preferential alignment of the fibers along any direction provides the material with an anisotropic structure, which can be described by the probability distribution function for fiber orientation, by orientation tensors, or at least using scalar orientation parameters, such as the orientation number:

$\eta_{\theta, i}=\frac{1}{N_{f}} \sum_{n=1}^{N_{f}} \cos \theta_{n}$

where $N_{f}$ is the number of fibers crossing the surface normal to the $i$-axis and $\cos \theta_{n}$ is the projection along the $i$-axis of a unit vector collinear with the $n^{\text {th }}$ fiber.

\section{NDT BASED ON THE MAGNETIC PROPERTIES OF THE FIBERS}

\subsection{Fundamentals}

In a previous work by Nunes et al [4] an inductor was developed consisting of a U- 
shaped ferrite core with copper wire coil around the two legs (see Figure 1). When an electric current is established through the copper winding a magnetic field is generated. The amount of magnetic flux $\Phi$ that is produced for a given electric current is defined as the inductance, $L$, which can be measured using an LCR meter. If the U-shaped inductor is placed over a UHPFRC layer, the inductance of the magnetic circuit depends on the magnetic permeability of the composite. As the relative permeability of the cementitious matrix is equal to 1.0 , irrespective of its composition and age, the relative permeability of the composite, and so the measured inductance, is governed by the properties of the fibers, namely the relative magnetic permeability of the fiber steel, the fiber content and the fiber orientation.

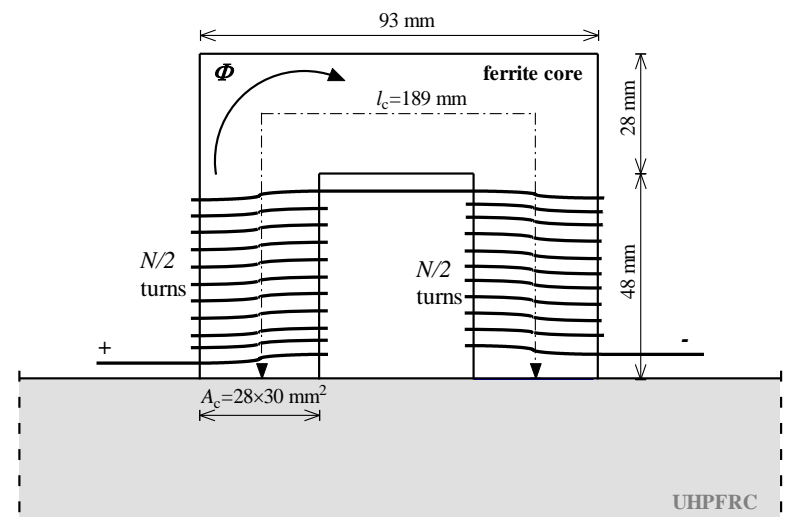

Figure 1: Schematic representation of the inductor placed over a UHPFRC layer.

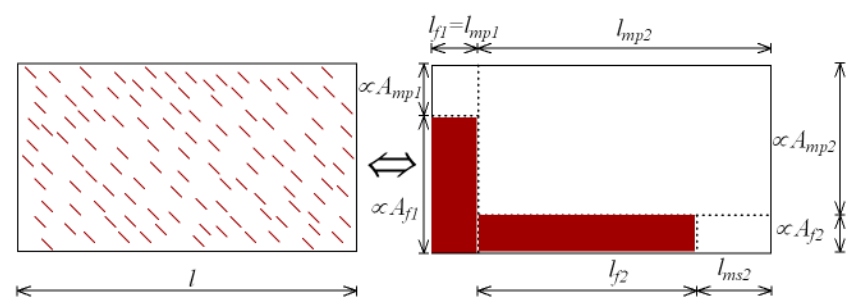

Figure 2: Equivalent physical model of UHPFRC.

In order to explain the inductance measurements obtained with the probe placed over an UHPFRC layer incorporating ferromagnetic fibers and providing the theoretical justification for the fiber content and fiber orientation indicators that are going to be defined later on, an electrical equivalent model has been established based on Hopkinson's law for magnetic circuits. The UHPFRC layer shown in Figure 1 is represented by a simplified physical model having all fibers aligned in the same direction and with negligible interaction between them, as shown in Figure 2.

Assuming that the fibers form an angle $\theta_{i}$ with the measuring direction, an equivalent system can be considered by dividing the total volume of fibers into two parts:

- the part corresponding to the projection of the fibers perpendicularly to the measuring direction having a volume fraction $V_{f 1}=V_{f} \sin ^{2} \theta_{i}$;

- plus the part corresponding to the projection of the fibers parallel with the measuring direction having a volume fraction of $V_{f 2}=V_{f} \cos ^{2} \theta_{i}$

in such a way that $V_{f 1}+V_{f 2}=V_{f}$. The corresponding magnetic circuit can thus be modelled as shown in Figure 3 , where $\mathcal{R}_{c}$ is the reluctance of the ferrite core of the magnetic probe; $\mathscr{R}_{\mathrm{f} 1}$ and $\mathscr{R}_{\mathrm{f} 2}$ are the equivalent magnetic reluctances of the fiber material fractions 1 and 2 , respectively; $\mathcal{R}_{\mathrm{mp} 1}$ corresponds to the magnetic reluctance of the matrix components in parallel with fiber material fraction $1 ; \mathcal{R}_{\mathrm{ms} 2}$ and $\Re_{\mathrm{mp} 2}$ correspond to the magnetic reluctance of the matrix components in series and in parallel with the fiber material fraction 2 , respectively.

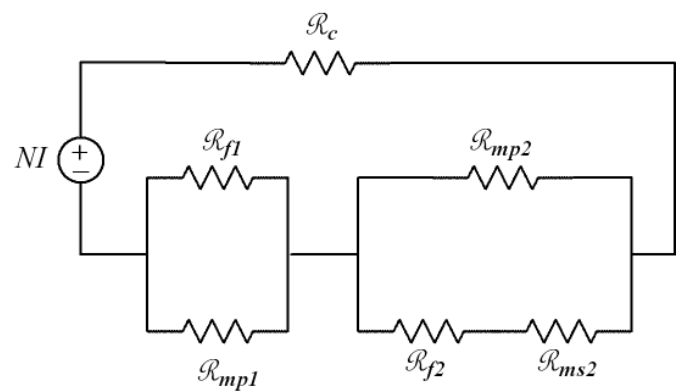

Figure 3: Electrical equivalent of the modelled magnetic circuit.

The inductance $L_{i}$ of the above modelled circuit becomes [4]:

$L_{i}=\frac{N^{2} \cdot \mu_{0}}{\frac{l_{c}}{\mu_{r, c} \cdot A_{c}}+\frac{l}{\mu_{r, i} \cdot A}}$ 
where $N$ is the number of turns in the copper coil; $\mu_{0}$ is the magnetic permeability of vacuum; $l_{c}$ and $l$ are the lengths of the magnetic path through the U-shaped core and UHPFRC layer, respectively; $A_{c}$ and $A$ are the corresponding cross-sectional areas; $\mu_{r c}$ is the relative magnetic permeability of the core material and $\mu_{r i}$ is the relative magnetic permeability of the composite, which has been shown [4] to be given by:

$\mu_{r, i}=\left(\frac{V_{f}^{*} \cdot \sin ^{2} \theta_{i}}{\gamma+\mu_{r, f} \cdot(1-\gamma)}+\right.$

$\left.\frac{\left(1-V_{f}^{*} \cdot \sin ^{2} \theta_{i}\right) \cdot\left(\mu_{r, f} \cdot \gamma+1-\gamma\right)}{\mu_{r, f} \cdot V_{f}^{*} \cdot \cos ^{2} \theta_{i}+\left(1-V_{f}^{*} \cdot \cos ^{2} \theta_{i}\right) \cdot\left(\mu_{r, f} \cdot \gamma+1-\gamma\right)}\right)^{-1}$

In the equation above, $\gamma$ is a model parameter expressing the fact that the fibers are discontinuous segments and not continuous steel filaments, $\mu_{r f}$ is the relative magnetic permeability of the fiber material and $V_{f}^{*}=(1-$ $\gamma) \cdot V_{f}$, where $V_{f}$ is the volumetric fiber fraction.

It has also been shown that, in the case of the developed inductor, the relative magnetic permeability of UHPFRC in a given direction $\mathrm{i}$ $\left(\mu_{r, i}\right)$ can be readily estimated from measured inductance values using the following approximation [4]:

$\mu_{r, i} \cong L_{i} / L_{\text {air }}$

where $L_{\text {air }}$ is the inductance measured placing the probe in the air away from any magnetic object.

\subsection{Determination of the fiber content}

The mean value of the relative magnetic permeability, $\mu_{r \text { mean }}$, obtained from measurements in two orthogonal directions $i$ and $j$, and given in Eq. (5), is practically independent of the fibre orientation and increases linearly with fibre content, see Figure 4.

$\mu_{r, \text { mean }}=0.5 \cdot\left(\mu_{r, i}+\mu_{r, j}\right)$

This is confirmed by the experimental data collected on laboratory specimens with welldefined fiber content and either with random or well oriented fibers [4], covering a wide range of fiber orientation distributions. For given material and geometry of the fibers, it is then possible to calibrate the linear relation between $\mu_{r \text {,mean }}$ and the volumetric fiber fraction $V_{f}$. The obtained regression line is independent of the matrix and represents a signature of the adopted fibers (or fiber mix).

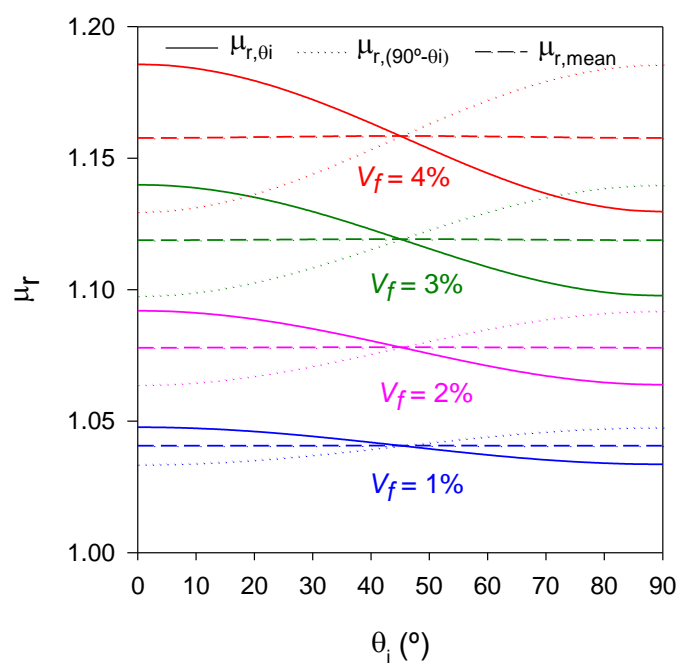

Figure 4: Variation of $\mu_{r, \theta_{i}}, \mu_{r,\left(90^{\circ}-\theta_{i}\right)}$ and $\mu_{r, \text { mean }}$ with the angle $\theta_{i}\left({ }^{\circ}\right)$.

In the case of the fiber mix adopted in the examples shown in this study, the obtained regression line is shown in Figure 5 and given by:

$$
\mu_{r, \text { mean }}=1.0+4.12 \cdot V_{f}
$$

The fiber mix is composed by two types of straight high strength steel fibers with lengths $l_{f}=9$ and $12 \mathrm{~mm}(50 \%$ each) and diameter $d_{f}=0.175 \mathrm{~mm}$.

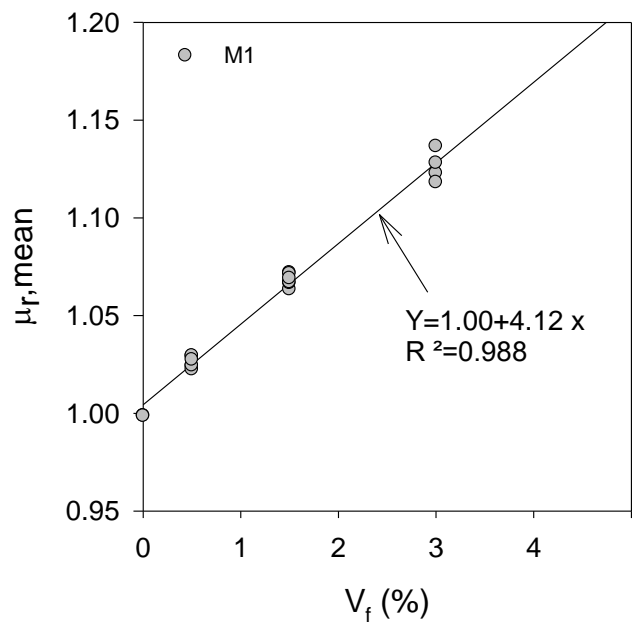

Figure 5: Calibration of a linear regression line relating $V_{f}$ and $\mu_{r, \text { mean }}$ for a fiber mix with $l_{f}=9$ and $12 \mathrm{~mm}(50 \%$ each) and $d_{f}=0.175 \mathrm{~mm}$. 


\subsection{Determination of the fiber orientation}

\section{factor}

From the same $\mu_{r, i}$ and $\mu_{r, j}$ values, the orientation indicator $\left(\rho_{i}-\rho_{j}\right)$, can be defined for identifying the direction of preferential orientation of the fibers and providing a scalar measure of the anisotropy in the fiber distribution with respect to the $i$-and $j$ directions:

$$
\left(\rho_{i}-\rho_{j}\right)=0.5 \frac{\mu_{r, i}-\mu_{r, j}}{\mu_{r, \text { mean }}-1}
$$

Based on the theoretical model outlined in section 2.1, this indicator was shown to be practically independent of the fiber content [4]. Positive values indicate orientation of the fibers along the $i$-direction while negative values indicate orientation of the fibers along the $j$-direction. Moreover, $\left(\rho_{i}-\rho_{j}\right)$, was found to vary linearly with $\cos ^{2} \theta$, as exemplified in Figure 6 assuming that all fibers have the same orientation (that is, the probability distribution of the fiber orientation angle is a Dirac delta).

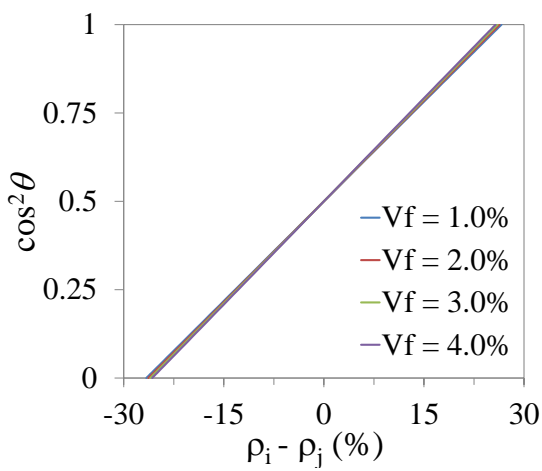

Figure 6: Predicted relation between the NDT orientation indicator $\left(\rho_{\mathrm{i}}-\rho_{j}\right)$ and $\cos ^{2} \theta$ (all fibers having the same orientation).

In case of real continuous fiber orientation distributions, it is interesting to confirm that the available experimental evidence indicates that $\left(\rho_{i}-\rho_{j}\right)$ and $\eta_{\theta, i}{ }^{2}$ are indeed linearly correlated, as shown in Figure 7. The dots correspond to experimental data obtained after image analysis of specimens with two fiber contents and a wide range of fiber orientation profiles obtained resorting to a magnetic orientation setup during casting. For details refer to [4][5][6].

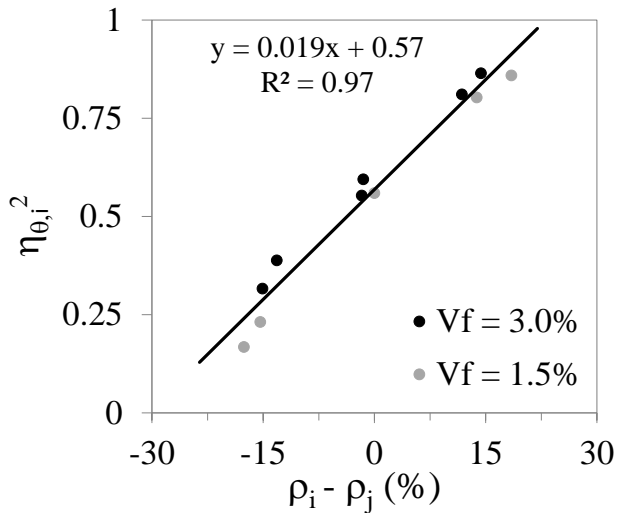

Figure 7: Observed linear correlation between NDT orientation indicator $\left(\rho_{i}-\rho_{j}\right)$ and the square orientation number determined by image analysis.

The fiber orientation factor $\alpha_{0, i}$ can be defined as the probability of a fiber to intersect a planar surface normal to the $i$-axis. It can be shown that is given by:

$\alpha_{0, i}=n_{f, i} \frac{A_{f}}{V_{f}}$

where $n_{f, i}$ is the number of fibers crossing a unit surface normal to the $i$-axis and $A_{f}$ is the cross-sectional area of a single fiber.

The same image analysis procedure was adopted to establish the relation between the orientation number $\eta_{\theta, i}$ and the orientation factor $\alpha_{0, i}$, as shown in Figure 8 [6]. The experimental data points suggest that:

$\alpha_{0, i} \approx \eta_{\theta, i}^{2}$

The dashed lines were determined using stereology principles, as explained in reference [6]. The 1D fiber distribution corresponds to a Dirac delta, while the 2D and 3D were determined assuming that both the in-plane and out-of-plane orientation angles are uniformly distributed within a certain range.

Accepting that $\alpha_{0, i}=\eta_{\theta, i}{ }^{2}$, the results shown in Figure 7 indicate that, in the case of the adopted fiber mix, the following linear relation can be used to determine the fiber orientation factor from the NDT orientation indicator [5][6]:

$\alpha_{0, i}=0.57+1.85 \cdot\left(\rho_{i}-\rho_{j}\right)$

As the orientation indicator defined by equation (10) is symmetric with respect to any 
two orthogonal directions $i$ and $j$, that is ( $\rho_{i}-$ $\left.\rho_{j}\right)=-\left(\rho_{j}-\rho_{i}\right)$, the fiber anisotropy is implied in this equation and is validated in Figure 9 with the available experimental evidence from references [1][2][7]. The experimental data points corresponds to the results from image analysis over two orthogonal planes perpendicular to the thickness in order to determine the corresponding fiber orientation factors. The thickness of the studied elements is comprised between 20 and $50 \mathrm{~mm}$ and the assumed casting direction was set to $\alpha_{0, j}$. The dashed lines were obtained according to Malena et al [3] and Nunes et al. [6] based on stereological principles. It is interesting to confirm that the fiber orientation factors estimated from equation (10) lie between the boundary cases determined using stereology for $2 \mathrm{D}$ and $3 \mathrm{D}$ fiber distributions and fit quite well the experimental data points.

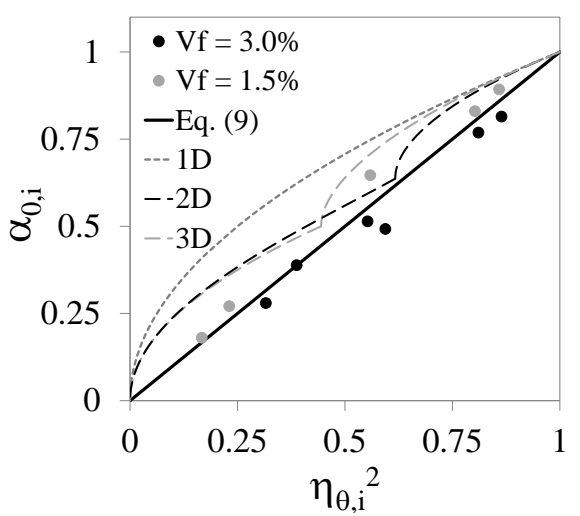

Figure 8: Relation between the square of the fiber orientation number and the fiber orientation factor.

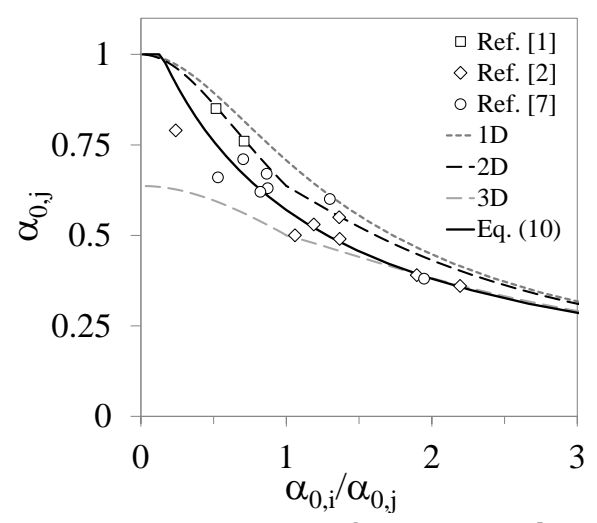

Figure 9: Orientation factors in orthogonal directions and comparison with experimental data from other authors.

\section{TENSILE STRENGTH OF UHPFRC}

\subsection{Physical model}

The tensile strength of UHPFRC, $f_{U t, u}$, is generally governed by fiber debonding followed by fiber pull-out, the latter marking the onset of the softening stage of the stressdisplacement curve. Considering a rigidplastic law for bond shear stress slip relation, and an average pull-out length equal to $l_{f} / 4$, it is possible to estimate $f_{U t, u}$ using equation (11):

$$
f_{U t, u, i}=\alpha_{0, i} \cdot \alpha_{1, i} \cdot \tau_{f} \cdot V_{f} \cdot \frac{l_{f}}{d_{f}}
$$

where the subscript $(\cdot)_{i}$ refers to the a direction perpendicular to the fracture surface, $\tau_{f}$ is the equivalent (rigid-plastic) bond strength at the fiber-to-matrix interface and $\alpha_{1, i}$ is the fiber efficiency factor, here defined as the as the expected value of the fiber efficiency function $g(\theta)$ :

$\alpha_{1, i}=\int_{0}^{\pi / 2} g(\theta) \cdot f_{i}(\theta) \cdot d \theta$

In the equation above, $f_{i}(\theta)$ is the probability density function (PDF) of the orientation angle of the fibers crossing the plane normal to the $i$ direction, $\theta\left(0^{\circ} \leq \theta \leq 90^{\circ}\right)$, and $g(\theta)$ is defined as the ratio between the pull-out force of a fiber oriented at an angle $\theta$ and the pull-out force of a perfectly aligned fiber $\left(\theta=0^{\circ}\right)$.

\subsection{Estimate of the fiber efficiency factor}

Following previous proposals, the efficiency function is here taken as $g(\theta)=1.0$ for $\theta \leq 60^{\circ}$. For orientation angles in the interval $60^{\circ}<\theta \leq 90^{\circ}$, the steel fiber efficiency is going to be neglected [8], in which case equation (12) becomes simply $\alpha_{1, i}=F_{i}\left(60^{\circ}\right)$, with $F_{i}(\theta)$ being the cumulative distribution function of $\theta$. This function can be established based on image analysis techniques. Laranjeira et al [9] concluded that the first and second moments of $\theta$ are correlated and can both be expressed as a function of the orientation number, $\eta_{\theta, i}$. The results from image analysis presented in [6] 
confirm this proposal and show that, in fact, it is possible to obtain good estimates of $\alpha_{1, i}$ as a function of $\eta_{\theta, i}\left(\right.$ or $\left.\alpha_{0, i}\right)$.

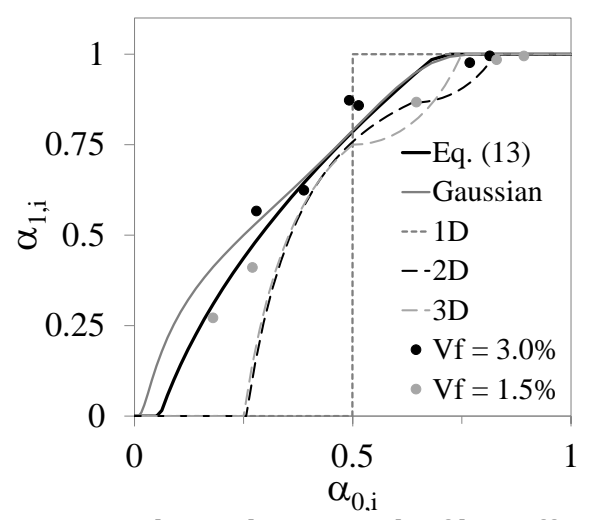

Figure 10: Relation between the fiber efficiency and orientation factors.

In the case of very favorable fiber orientation, only a negligible number of fibers is oriented at $\theta>60^{\circ}$ leading to $\alpha_{1} \approx 1.0$. For less favorable orientation scenarios, the image analysis results presented in [6] evidence a linear relation between $\alpha_{1}$ and $\eta_{\theta, i}$. Considering equation (9), this led to the following equation relating the fiber efficiency and orientation factors:

$$
\alpha_{1, i}=1.686 \cdot \sqrt{\alpha_{0, i}}-0.406,0 \leq \alpha_{1, i} \leq 1
$$

This equation is compared to experimental data in Figure 10. The experimental data points were determined using the empirical cumulative distribution of the angle $\theta$ and the corresponding $\alpha_{0, i}$, both determined from image analysis. The solid grey line corresponds to the $F_{i}\left(60^{\circ}\right)$ values that are obtained assuming a Gaussian distribution for $\theta$, with mean and standard deviation determined from $\eta_{\theta, i}$, according to Laranjeira et al [9]. The dashed lines were determined using the same stereological principles mentioned above and described in [3][6]. It can be seen that the proposed equation fits quite well the available data and, except for the cases of extremely unfavorable fiber orientation (i.e., $\alpha_{1, i}<0.25$ ), provides $\alpha_{1, i}$ values that are equivalent to those obtained assuming that $f_{i}(\theta)$ is a Gaussian PDF.

\subsection{Estimate of the equivalent fiber-to- matrix bond strength}

The fiber-to-matrix bond shear stress slip relation can be determined from pull-out tests. Alternatively, considering that $\tau_{f}$ is the remaining unknown, equation (11) indicates that $\tau_{f}$ can be determined from laboratory tensile characterization test results by fitting a line of the type $f_{U t, u}=\lambda \cdot \tau_{f}$ to the experimental data, as exemplified in Figure 11, where $\lambda=\alpha_{0} \cdot \alpha_{1} \cdot V_{f} l_{f} d_{f}$. The tensile characterization tests can either be direct tensile tests, or any other well established indirect test requiring inverse analysis procedures to determine the tensile strength. In the case of the presented 11 experimental data points, $\alpha_{0}$ and $\alpha_{1}$ were determined by image analysis of planes parallel to the fracture surface [6]. The Double-Edge Wedge-Splitting (DEWS) test developed by Di Prisco et al [10] was adopted for quantifying the corresponding tensile strength, and $\sigma_{N, \text { peak }}$ refers to the post-cracking peak tensile stress obtained during the DEWS tests. It has been shown [10][11] that $\sigma_{N, \text { peak }} \approx$ $f_{U t, u}$, either for strain-hardening and strainsoftening fiber-reinforced composites.

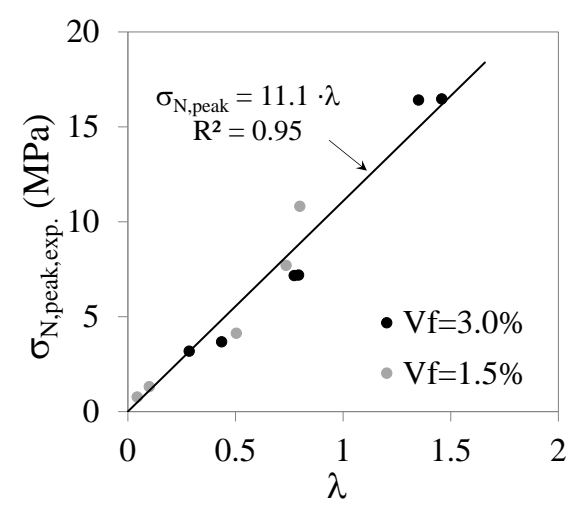

Figure 11: $\sigma_{\mathrm{N} \text {,peak }}$ experimental results plotted against $\lambda$ $=\alpha_{0} \cdot \alpha_{1} \cdot V_{f} l_{f} / d_{f}$ computed from image analysis.

The range of fiber orientations in the tested specimens should be wide enough to ensure the significance of the fitted line. As mentioned above, in the present case this has been achieved using a magnetic orientation setup during casting of the UHPFRC in the molds. The obtained value of $\tau_{f} \approx 11 \mathrm{MPa}$, is within the range of values proposed in 
reference [12] and depends both on the matrix and the fibers. For details regarding the adopted UHPFRC composition, refer to [11].

After estimating $\tau_{f}$, the NDT measurements together with the equations (10), (11) and (13) were used to predict the $\sigma_{\mathrm{N} \text {,peak }}$ values of 36 DEWS specimens. The comparison between the predicted and the experimental values is shown in Figure 12 and good agreement is found, illustrating the great potential of the developed procedure to assess the effect of fiber orientation on the tensile behavior of UHPFRC.

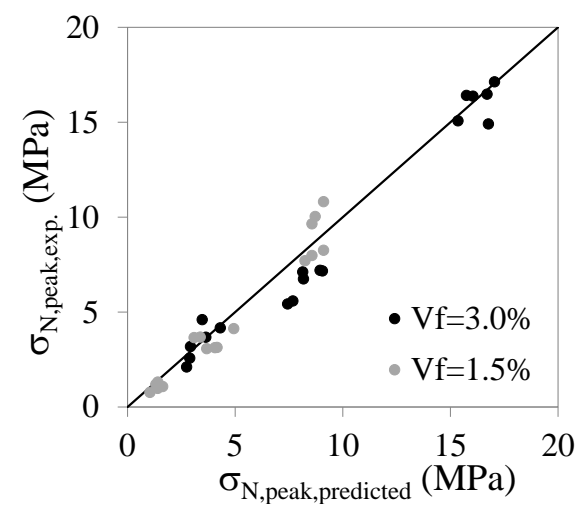

Figure 12: Comparison between the $36 \sigma_{\mathrm{N} \text {,peak values }}$ obtained experimentally and those predicted using the magnetic probe measurements and equations (10), (11) and (13).

\section{MODELLING OF THE TENSILE RESPONSE}

\subsection{General}

A simple meso-mechanical model is adopted for obtaining the representative tensile response of the UHPFRC as a function of volumetric fiber fraction and of the fiber orientation factor determined from the NDT measurements. The model is based on the works by Wuest [7], Oesterlee [2] and Pfyl [13]. The representative volume element (RVE) of the composite is assumed to contain several potential cracks, some of which are activated during the loading process, depending on the ratio between the cracking strength of the matrix, $f_{m t}$, and the tensile strength of the composite $f_{U t, u}$ as determined by the fibers and given by equation (11). The matrix cracking strength is assumed to vary within the RVE according to a Gaussian distribution. A crack is arises when the lowest matrix cracking stress in the RVE is reached. The corresponding cracking strength of the composite $f_{U t, e l}$ is estimated as a function of $f_{m t}$, $V_{f}$ and of the elasticity modulus of the fibers, $E_{f}$, and matrix, $E_{m}$ :

$$
f_{U t, e l}=\left[1+\alpha_{0, i} \cdot \alpha_{1, i} \cdot\left(\frac{E_{f}}{E_{m}}-1\right) \cdot V_{f}\right] \cdot f_{t m}
$$

\subsection{Tensile response of a single crack}

The tensile response of an active crack is obtained from the corresponding crack opening, $w$, by summing the contributions of the matrix, $\sigma_{b r i}$, fiber pre-stress, $\sigma_{p r e}$, and fiber debonding followed by pullout, $\sigma_{f}$ :

$\sigma(w)=\sigma_{b r i}+\sigma_{p r e}+\sigma_{f}$

An exponential law is adopted for the cracking bridging stresses transferred by the matrix:

$$
\frac{\sigma_{b r i}}{f_{t m}}=\exp \left(-\frac{f_{t m}}{G_{F m}} \cdot w\right)
$$

where $\mathrm{G}_{F m}$ is the matrix fracture energy. The fiber prestress mobilized prior to cracking during the elastic deformation of the fibers is assumed to be released according to a linear law:

$$
\frac{\sigma_{p r e}}{f_{t m}}=\alpha_{0, i} \cdot \alpha_{1, i} \cdot\left(\frac{E_{f}}{E_{m}}-1\right) \cdot V_{f} \cdot \frac{w_{d e b}-w}{w_{d e b}}
$$

with the full fiber prestress release occurring when the fibers are fully debonded, that is, when $w=w_{d e b}$ :

$w_{d e b}=\frac{\tau_{f} \cdot l_{f}^{2}}{E_{f} \cdot d_{f}}$

The crack bridging stresses due to all the fibers crossing the fracture surface are defined according to Pfyl [13] during both the debonding $\left(w \leq w_{d e b}\right)$ and the pullout stages $\left(w>w_{d e b}\right)$ : 


$$
\begin{aligned}
& \frac{\sigma_{f}}{f_{U t, u}}=2 \cdot \sqrt{\frac{w}{w_{d e b}}}-\frac{w}{w_{d e b}} \quad, w \leq w_{d e b} \\
& \frac{\sigma_{f}}{f_{U t, u}}=\left(1-2 \cdot \frac{w-w_{d e b}}{l_{f}}\right)^{2}, w>w_{d e b}
\end{aligned}
$$

\subsection{Multi-crack model for strain hardening composites}

The matrix cracking strength is randomly attributed to each potential crack in the RVE according to a Gaussian distribution. Two conditions must be simultaneously fulfilled for the formation of a new crack: 1) the acting tensile stress must exceed the composite cracking stress $f_{U t, e l}$ in one of the potential cracks 2) the distance between two adjoining cracks must be larger than the transfer length. The latter is approximated by:

$s_{r, \text { min }}=\frac{0.25}{\alpha_{0, i} \cdot \alpha_{2, i}} \frac{f_{U t, e l}}{\tau_{f}} \frac{d_{f}}{V_{f}}$

where $\alpha_{2, i}$ is a coefficient that takes into account that the fibers are not continuous filaments. In this work it has been set to 0.4.

The tensile response of the composite is obtained by conducting a force driven analysis up to $f_{U t, u}$ after which a crack opening displacement (COD) controlled analysis is made considering that the deformations are localized in one of the active cracks while the others unload.

\subsection{Examples}

Examples of tensile stress-strain curves calculated for the UHPFRC mix adopted in this study are shown in Figure 13 as a function of the fiber orientation factor $\alpha_{0}$. The corresponding fiber efficiency factor has been determined using equation (13). The equivalent rigid-plastic fiber-to-matrix bond strength $\tau_{f}=11 \mathrm{MPa}$ has been adopted, according to section 3.3. The fiber orientation factor $\alpha_{0}=0.50$ is representative of a 3D random distribution of fibers, in which case the material can be considered isotropic. According to equation (10) the value $\alpha_{0}=0.57$ is representative of thin UHPFRC layers with non-oriented fibers with respect to any of the in-plane directions. The $\alpha_{0, i}=0.85$ is representative of a UHPFRC layer with a strong orientation of the fibers along the $i$ direction. According to equation (10) and Figure 9, the orientation factor in the perpendicular direction is $\alpha_{0, j}=0.29$, in which case the material exhibits a markedly anisotropic behavior.

The remaining model parameters have been tentatively set as: $E_{m}=40 \mathrm{GPa}, G_{F m}=20 \mathrm{~N} / \mathrm{m}$, mean value of the matrix cracking strength, $f_{m t, m}=11 \mathrm{MPa}$ (i.e., $f_{m t, m} / \tau_{f}=1$ ) and $\operatorname{COV}\left(f_{m t}\right)=0.12$.

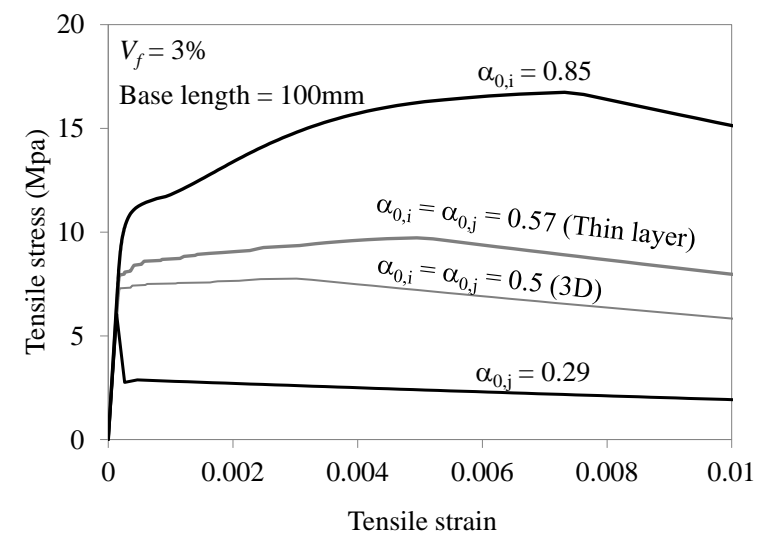

a)

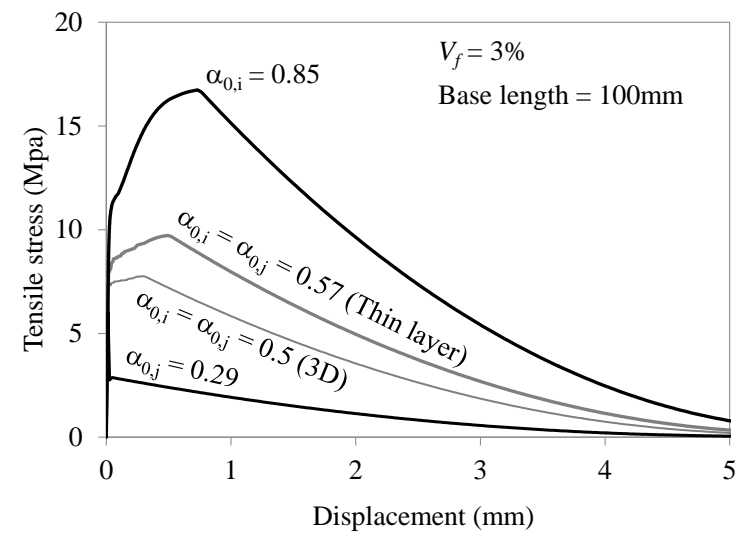

Figure 13: Tensile constitutive laws for the adopted UHPFRC as a function of the fiber orientation factor.

\section{APPLICATION - CANTILEVER SLAB STRIPS}

\subsection{Geometry of the specimens}

As part of a broader experimental campaign described in detail in reference [14], 400mm wide reinforced concrete (RC) slab strips 
strengthened with a $50 \mathrm{~mm}$ thick UHPFRC layer were tested to failure. The UHFRC layer is either unreinforced $(\mathrm{U})$ or reinforced with ribbed bars (RU). The elements described herein all failed in bending. The geometry of the tested elements with the UHPFRC layer is defined in Figure 14.
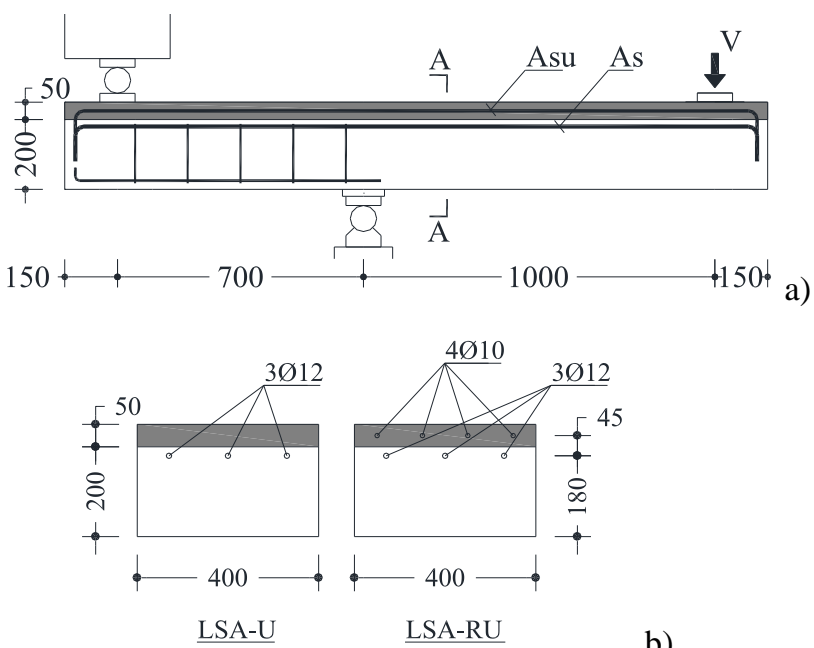

Figure 14: Geometry of the tested beams: a) elevation; b) cross-sections and specimen designation. Conventional concrete in white and the UHPFRC layer in dark grey.

Also non-strengthened (reference) specimens were tested. These specimens are designated as LSA, are $215 \mathrm{~mm}$ deep and contain only the reinforcements embedded in the RC zone. Additionally, slab strips with the same total depth $(250 \mathrm{~mm})$ and reinforcements as the strengthened specimens were tested. These are designated by LDA. In order to ensure representativeness of the results two beams of each configuration were tested, in total 8 experiments.

The conventional concrete presented a uniaxial compressive strength $f_{c}=35 \mathrm{MPa}$ and a maximum aggregate size $d_{g}=14 \mathrm{~mm}$. The reinforcement steel belongs to the strength class S500 and can be classified as highductility steel. For details refer to [14].

\subsection{NDT measurements}

Some heterogeneity of fiber orientation is expected in the strengthening layers due to the adopted casting procedure. The NDT measurements performed on one of the slab strips LSA-U will be taken as illustrative example showing how the proposed methodology can be used to predict the tensile behavior of the UHPFRC strengthening layer in such circumstances.

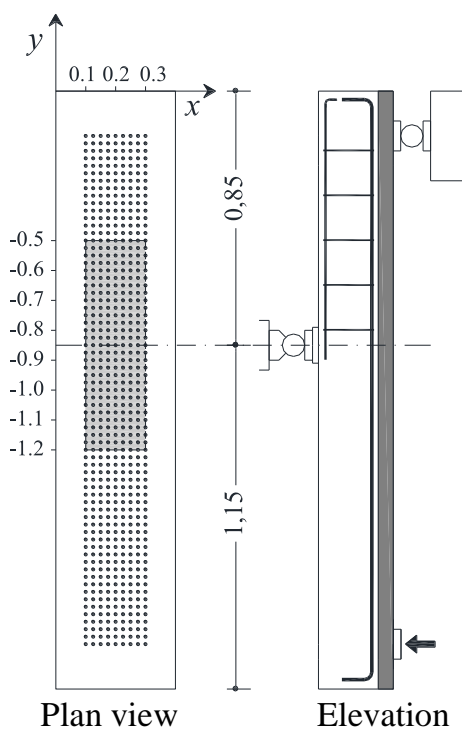

Figure 15: Grid for NDT measurements. The shaded area corresponds to the area were the results of the measurements are presented in the figures below.
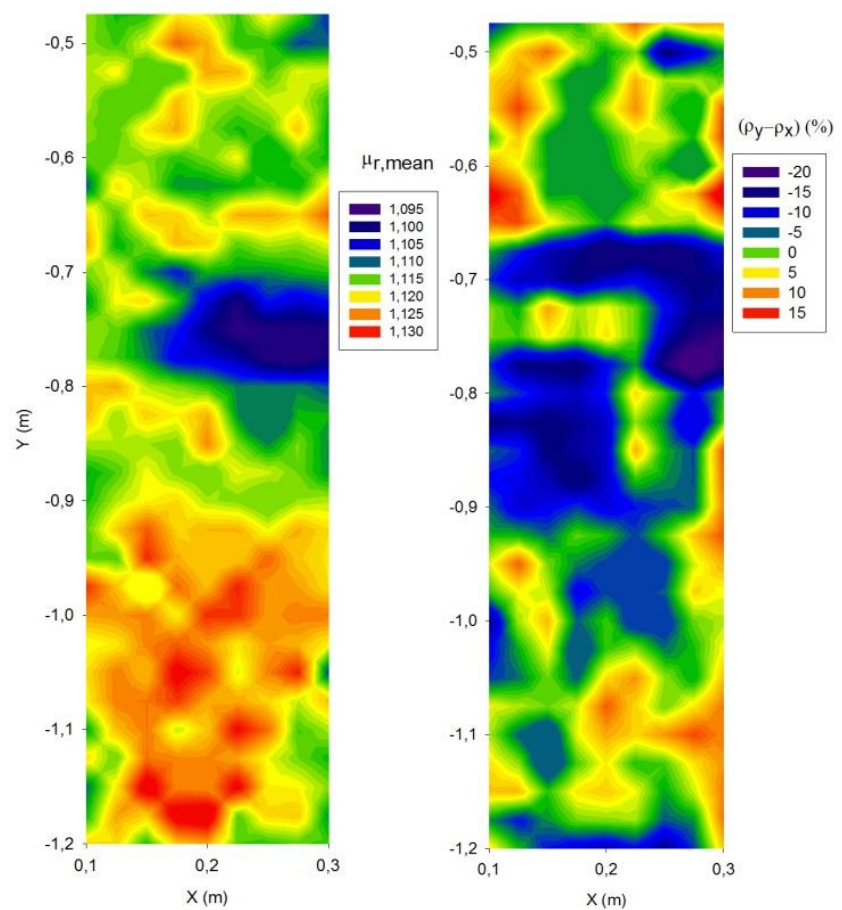

Figure 16: Contour maps with the $\mu_{r, \text { mean }}$ and $\left(\rho_{Y}-\rho_{X}\right)$ indicators obtained by NDT.

Two orthogonal inductance measurements along the $X$ - and $Y$-directions were taken at each of the grid points identified in Figure 15. The spacing of the measuring points is $25 \mathrm{~mm}$ 
in the $\mathrm{x}$ - and $\mathrm{y}$-directions. The $\mu_{r, \text { mean }}$ and $\left(\rho_{Y^{-}}\right.$ $\rho_{X}$ ) indicators given by equations (5) and (7) are presented in Figure 16. The area over which the results are plotted is that identified by the grey solid hatch in Figure 15 . The support alignment is located at $\mathrm{y}=-0.85 \mathrm{~m}$. The $\mu_{r \text {,mean }}$ values confirm that there is some heterogeneity in the fiber distribution along the layer. The $\left(\rho_{Y^{-}} \rho_{X}\right)$ values clearly indicate that there is unfavorable fiber orientation in the dark blue areas.

\subsection{Estimate of the tensile constitutive laws}

The tensile strength of the UHPFRC was estimated using equation (11), with the parameters $V_{f}, \alpha_{0}$ and $\alpha_{1}$ and being determined from the NDT measurements illustrated in Figure 16 and using equations (6), (10) and (13), respectively. The corresponding contour map with the computed values of $f_{U t, u}$ at each point is shown in Figure 17 a). The regions identified by the dark blue colour indicate that the tensile strength of the material is clearly below the average.

As a plane stress model is adopted in the next section to model the structural behaviour of the slab strips, the tensile strength of the UHPFR was averaged across the $\mathrm{x}$-direction so that a single $f_{U t, u}$ value could be assigned to each $\mathrm{y}$-coordinate. The obtained results are presented in Figure 17 b) by the blue dots. The red line indicates the tensile strength adopted in the numerical model. The tensile-stress strain curves for each zone were defined using the meso-mechanical model described in section 4 and used as input in the structural finite element model.

\subsection{Numerical model for structural analysis}

A 2D smeared fixed crack model based on a total strain concept currently implemented in the finite element code DIANA 9.5 was adopted for modelling the tested beams. For details refer to [15]. The finite element mesh is depicted in Figure 18. Each element is $5 \times 5 \mathrm{~cm} 2$ and the crack band width has been taken as $h=5 \mathrm{~cm}$. Eight nodded elements plane stress with $2 \times 2$ Gaussian integration scheme have been adopted. The reinforcements are modelled using an embedded formulation in which the displacements of the reinforcement truss elements are interpolated from those of the embedding concrete elements. Interface elements have been placed in between the ordinary concrete and the UHPFRC finite elements. The interface behaves as infinitely stiff until a tensile vertical tensile stress of 1.0MPa, after which null shear and normal stiffness is assumed.

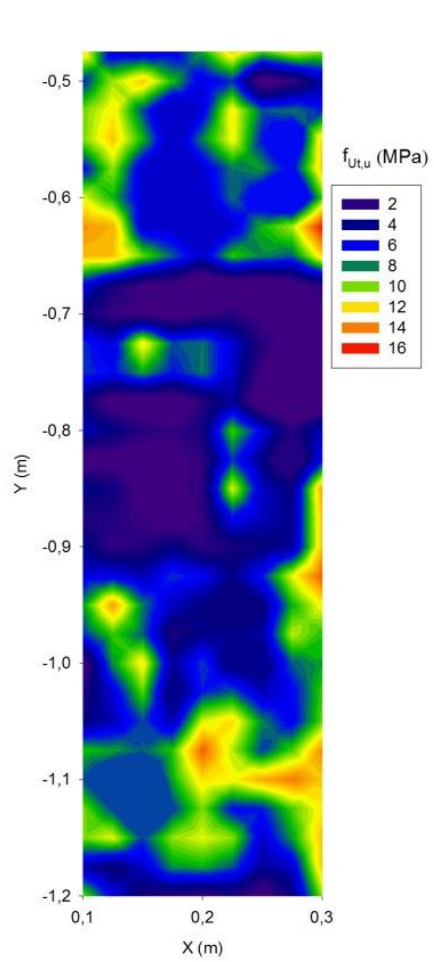

a)

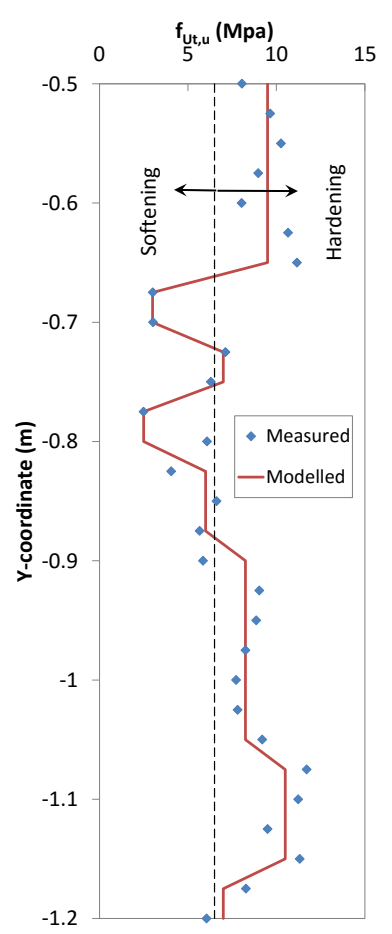

b)
Figure 17: Variation of the uniaxial tensile strength of the UHPFRC, $f_{U t, u}$ : a) Contour levels; b) variation along the $\mathrm{Y}$-axis of the $f_{U t, u}$ values averaged across the $\mathrm{X}$ direction.

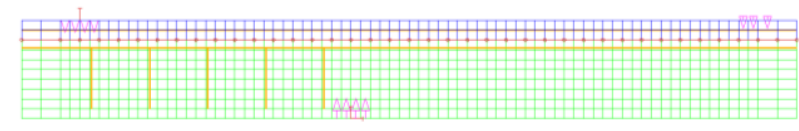

Figure 18: Finite element mesh of slab-strips. Interface elements represented by the red line. Dark blue and light green are the UHPFRC and ordinary concrete elements, respectively. The orange lines represent the reinforcement bars.

\subsection{Results}

The comparison between the calculated and the experimental force-displacement curves is shown in Figure 19. The vertical displacement $\delta_{V, 1}$ refers to the section were the force $\mathrm{V}$ is 
applied. The light grey lines correspond to the reference specimens LSA and LDA without UHPFRC layer. The red lines correspond to the numerical simulations considering the ideal scenario of uniform 3D fiber distribution $\left(\alpha_{0, i}=0.5\right)$. It can be seen that the calculated curves fit quite well those of the LSA-RU beam containing reinforcement bars in the UHPFRC layer. Both in the experiment and in the numerical simulation failure occurred due to rebar failure in the UHPFRC layer.

However, the assumption of uniform fiber distribution leads to a quite poor fit of the behavior of the LSA-U beam without reinforcing bars in the UHPFRC layer. The strength of the beam is overestimated by a large margin. The blue line corresponds to the numerical simulation performed using the tensile strength values determined from the NDT measurements according to Figure $17 \mathrm{~b}$ ). It can be seen that the agreement is excellent, which provides further confirmation of the adequacy of the procedure proposed for estimating the tensile behavior of UHPFRC.

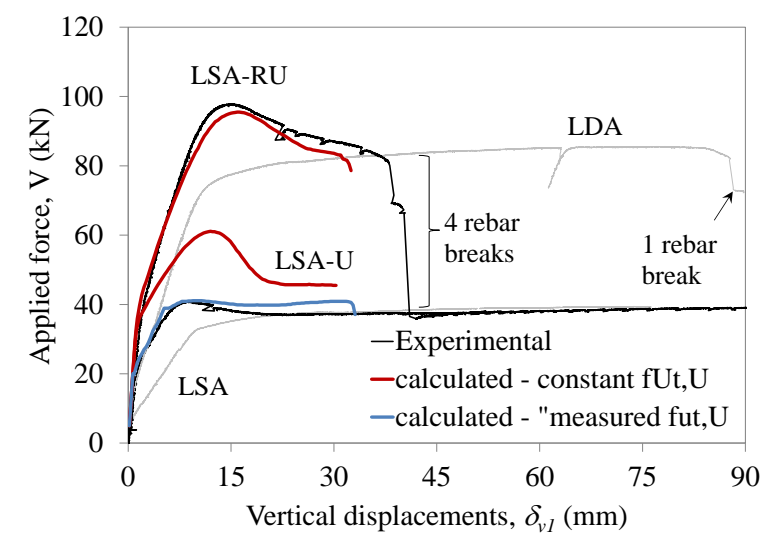

Figure 19: Force-displacement. The constant $f_{t U, t}$ scenario corresponds to $\alpha_{0, i}=0.5$ everywhere throughout the UHPFRC layer.

\section{CONCLUSIONS}

A procedure is proposed for estimating the constitutive law of UHPFRC based on magnetic inductance measurements along two orthogonal directions. It was shown how two scalar indicators obtained from these measurements can be correlated to the fiber content $V_{f}$ and fiber orientation factor $\alpha_{0, i}$. Jointly with the fiber efficiency factor $\alpha_{1, i}$, which can be obtained as a function of $\alpha_{0, i}$, these parameters can be used to predict the tensile strength of the UHPFRC when governed by fiber pullout. In the absence of a complete tensile characterization of the material covering a wide range of fiber orientations and dosages, the full tensile stressstrain curve of the material can be determined using a meso-mechanical model of the composite, as that described in section 4 .

For a new UHPFRC mix, the implementtation of this procedure requires the following laboratory work:

1) determination of $\tau_{f}$ by means of pull-out tests or using the method outlined in section 3.3;

2) calibration of a regression line relating $V_{f}$ with the mean relative magnetic permeability $\left(\mu_{\mathrm{r}, \text { mean }}\right)$ as described in section 2.2;

3) calibration of the regression line relating $\alpha_{0}$ with the orientation indicator $\left(\rho_{i}-\rho_{j}\right)$ as described in section 2.3.

In case the UHPFRC matrix is changed, keeping the fibers mix, only step 1) needs to be repeated, since the regression lines calibrated in steps 2) and 3) are a signature of the fibers.

It was also evidenced by the results presented in section 5 that the structural behavior of UHPFRC layers containing reinforcement bars is not influenced by local fiber content and orientation variations. In this case, the force deformation curves obtained in the experiments could be accurately reproduced considering the tensile constitutive law of the UHPFRC corresponding to a uniform distribution of fibers. However, in the case of the layers without reinforcement bars, the behavior could only be reproduced using the tensile constitutive laws estimated from the NDT measurements and taking into account the spatial variability.

\section{ACKNOWLEDGMENTS}

This research was financed by FEDER funds under the Operational Program Factors of Competiveness - COMPETE and by National Funds under FCT - Foundation for 
Science and Technology through project PTDC/ECM/122446/2010 - Engineered high performance fibre reinforced concrete materials: de-sign, characterization and quality control. Collaboration and materials supply by STAP, Concremat, Secil, Omya Comital, Sika, and KrampeHarex is gratefully acknowledged.

\section{REFERENCES}

[1] Ferrara L, Ozyurt N, Prisco M. 2011. High mechanical performance of fibre reinforced cementitious composites: the role of 'casting-flow induced' fibre orientation. Materials \& Structures, 44:109-28.

[2] Oesterlee C. 2010. Structural Response of Reinforced UHPFRC and RC Composite Members. Doctoral thesis. École Polytechnique et Fédérale de Lausanne.

[3] Bastien-Masse M, Denarié E, Brühwiler E. 2016. Effect of fiber orientation on the in-plane tensile response of UHPFRC reinforcement layers. Cement \& Concrete Composites, 67:111-125.

[4] Nunes, S., Pimentel, M. \& Carvalho, A. 2016. Non-destructive assessment of fibre content and orientation in UHPFRC layers based on a magnetic method. (Submitted to Cement \& Concrete Composites)

[5] Nunes, S., Pimentel, M. and Carvalho, A. 2016. Non-Destructive Assessment of Fibre Content and Orientation in UHPFRC Reinforcement Layers. In HiPerMat 2016, Proceedings of the 4th International Symposium on UHPC and High Performance Construction Materials, Kassel.

[6] Nunes, S., Pimentel, M., MilheiroOliveira, P. Carvalho, A. 2016. Estimation of the tensile strength of UHPFRC layers based on non-destructive assessment of the fibre content and orientation (Submitted to Cement \& Concrete Composites)

[7] Wuest J. 2007. Comportement structural des bétons de fibres ultra performants en traction dans des éléments composés. Doctoral thesis. École Polytechnique et Fédérale de Lausanne.
[8] Foster S. J. 2001. On Behavior of HighStrength Concrete Columns: Cover Spalling, Steel Fibers, and Ductility. ACI Structural Journal. 98:583-589.

[9] Laranjeira F, Grünewald S, Walraven J, Blom C, Molins C, Aguado A. 2011. Characterization of the orientation profile of steel fiber reinforced concrete. Materials \& Structures; 44:1093-111.

[10] Di Prisco, M., Ferrara L, Lamperti M.G.L. 2013. Double edge wedge splitting (DEWS): an indirect tension test to identify post-cracking behaviour of fibre reinforced cementitious composites. Materials \& Structures, 46:1893-1918.

[11] Pimentel, M., Nunes, S. 2016. On the Double-Edge Wedge-Splitting Test for the Characterization of HPFRC in Uniaxial Tension. In HiPerMat 2016, Proceedings of the 4th International Symposium on UHPC and High Performance Construction Materials, Kassel.

[12] Wille K, El-Tawil S, Naaman, A. 2013. Properties of Strain Hardening Ultra High Performance Fiber Reinforced Concrete (UHP-FRC) under Direct Tensile Loading. Cement \& Concrete Composites; 48:53-66.

[13] Pfyl T. 2003. Tragverhalten von Stahlfaserbeton. Doctoral Thesis No. 15005. ETH Zurich.

[14] Pimentel, M., Nunes, S. 2016. Experimental Tests On RC Beams Reinforced With a UHPFRC Layer Failing in Bending and Shear. In HiPerMat 2016, Proceedings of the 4th International Symposium on UHPC and High Performance Construction Materials, Kassel.

[15] Pimentel, M., Nunes, S. 2016. Determination of the fibre content and orientation in UHPFRC layers using NDT - application to the simulation of the behaviour of strengthened beams. In IABMAS 2016, Proceedings of the 8th International Conference on Bridge Maintenance, Safety and Management, Foz do Iguaçu, Brazil 